\title{
Quasi-greedy bases and Lebesgue-type inequalities
}

\author{
S. J. Dilworth \\ Department of Mathematics, University of South Carolina \\ Columbia, SC 29208, USA \\ E-mail: dilworth@math.sc.edu \\ M. Soto-Bajo \\ Departamento de Matemáticas, Facultad de Ciencias, Universidad Autónoma de Madrid \\ Cantoblanco, carretera de Colmenar Km 15, Madrid, Spain \\ E-mail: moises.soto@uam.es \\ V. N. Temlyakov \\ Department of Mathematics, University of South Carolina \\ Columbia, SC 29208, USA \\ E-mail: temlyakovusc@gmail.com
}

\begin{abstract}
We study Lebesgue-type inequalities for greedy approximation with respect to quasi-greedy bases. We mostly concentrate on this study in the $L_{p}$ spaces. The novelty of the paper is in obtaining better Lebesgue-type inequalities under extra assumptions on a quasigreedy basis than known Lebesgue-type inequalities for quasi-greedy bases. We consider uniformly bounded quasi-greedy bases of $L_{p}$, $1<p<\infty$, and prove that for such bases an extra multiplier in the Lebesgue-type inequality can be taken as $C(p) \ln (m+1)$. The known magnitude of the corresponding multiplier for general (no assumption of uniform boundedness) quasi-greedy bases is of order $m^{\left|\frac{1}{2}-\frac{1}{p}\right|}$, $p \neq 2$. For uniformly bounded orthonormal quasi-greedy bases we get further improvements replacing $\ln (m+1)$ by $(\ln (m+1))^{1 / 2}$.
\end{abstract}

2010 Mathematics Subject Classification: Primary 41A65; Secondary 41A25, 41A46, $46 \mathrm{~B} 20$. 


\section{Introduction}

We study the efficiency of greedy algorithms for $m$-term nonlinear approximation with regard to bases. Our primary interest is in approximation in $L_{p}$ with respect to quasi-greedy bases. Let $X$ be an infinite-dimensional separable Banach space with a norm $\|\cdot\|:=\|\cdot\|_{X}$ and let $\Psi:=\left\{\psi_{k}\right\}_{k=1}^{\infty}$ be a semi-normalized basis for $X\left(0<c_{0} \leq\left\|\psi_{k}\right\| \leq C_{0}, k \in \mathbb{N}\right)$. All bases considered in our paper are assumed to be semi-normalized. For a given $f \in X$ we define the best $m$-term approximation with regard to $\Psi$ as follows:

$$
\sigma_{m}(f):=\sigma_{m}(f, \Psi)_{X}:=\inf _{b_{k}, \Lambda}\left\|f-\sum_{k \in \Lambda} b_{k} \psi_{k}\right\|_{X}
$$

where the infimum is taken over coefficients $b_{k}$ and sets $\Lambda$ of indices with cardinality $|\Lambda|=m$. There is a natural algorithm of constructing an $m$-term approximant. For a given element $f \in X$ we consider the expansion

$$
f=\sum_{k=1}^{\infty} c_{k}(f) \psi_{k}
$$

We call a permutation $\rho, \rho(j)=k_{j}, j=1,2, \ldots$, of the positive integers decreasing and write $\rho \in D(f)$ if

$$
\left|c_{k_{1}}(f)\right| \geq\left|c_{k_{2}}(f)\right| \geq \ldots
$$

In the case of strict inequalities here $D(f)$ consists of only one permutation. We define the $m$-th greedy approximant of $f$ with regard to the basis $\Psi$ corresponding to a permutation $\rho \in D(f)$ by formula

$$
G_{m}(f):=G_{m}(f, \Psi):=G_{m}(f, \Psi, \rho):=\sum_{j=1}^{m} c_{k_{j}}(f) \psi_{k_{j}} .
$$

This algorithm is known in the theory of nonlinear approximation under the name of Thresholding Greedy Algorithm (TGA). The best we can achieve with the algorithm $G_{m}$ is

$$
\left\|f-G_{m}(f)\right\|_{X}=\sigma_{m}(f, \Psi)_{X}
$$

or a little weaker

$$
\left\|f-G_{m}(f)\right\|_{X} \leq C \sigma_{m}(f, \Psi)_{X}
$$

for all $f \in X$ with a constant $C$ independent of $f$ and $m$. The following concept of a greedy basis has been introduced in [9]. 
Definition 1.1. We call a basis $\Psi$ a greedy basis if for every $f \in X$ there exists a permutation $\rho \in D(f)$ such that

$$
\left\|f-G_{m}(f, \Psi, \rho)\right\|_{X} \leq C \sigma_{m}(f, \Psi)_{X}
$$

with a constant $C$ independent of $f$ and $m$.

We refer the reader to a survey [24] and a book [25] for further discussion of greedy type bases. In this paper we are interested in special inequalities - Lebesgue-type inequalities - for greedy approximation.

Lebesgue [12] proved the following inequality: for any $2 \pi$-periodic continuous function $f$ we have

$$
\left\|f-S_{n}(f)\right\|_{\infty} \leq\left(4+\frac{4}{\pi^{2}} \ln n\right) E_{n}(f)_{\infty},
$$

where $S_{n}(f)$ is the $n$th partial sum of the Fourier series of $f$ and $E_{n}(f)_{\infty}$ is the error of the best approximation of $f$ by the trigonometric polynomials of order $n$ in the uniform norm $\|\cdot\|_{\infty}$. The inequality (1.1) relates the error of a particular method $\left(S_{n}\right)$ of approximation by the trigonometric polynomials of order $n$ to the best-possible error $E_{n}(f)_{\infty}$ of approximation by the trigonometric polynomials of order $n$. By the Lebesgue-type inequality we mean an inequality that provides an upper estimate for the error of a particular method of approximation of $f$ by elements of a special form, say, form $\mathcal{A}$, by the best-possible approximation of $f$ by elements of the form $\mathcal{A}$. In the case of approximation with regard to bases (or minimal systems), the Lebesgue-type inequalities are known both in linear and in nonlinear settings (see surveys [10], [23], and [24]).

By the Definition 1.1 greedy bases are those for which we have ideal (up to a multiplicative constant) Lebesgue-type inequalities for greedy approximation. In this paper we concentrate on a wider class of bases than greedy bases - quasi-greedy bases. The concept of quasi-greedy basis was introduced in [9].

Definition 1.2. The basis $\Psi$ is called quasi-greedy if there exists some constant $C$ such that

$$
\sup _{m}\left\|G_{m}(f, \Psi)\right\| \leq C\|f\| .
$$

Subsequently, Wojtaszczyk [28] proved that these are precisely the bases for which the TGA merely converges, i.e.,

$$
\lim _{n \rightarrow \infty} G_{n}(f)=f .
$$

The main result of [27] is the following Lebesgue-type inequality for greedy approximation with respect to a quasi-greedy basis in the $L_{p}$ spaces. 
Theorem 1.1. Let $1<p<\infty, p \neq 2$, and let $\Psi$ be a quasi-greedy basis of the $L_{p}$ space. Then for each $f \in L_{p}$ we have

$$
\left\|f-G_{m}(f, \Psi)\right\|_{L_{p}} \leq C(p, \Psi) m^{|1 / 2-1 / p|} \sigma_{m}(f, \Psi)_{L_{p}}
$$

Theorem 1.1 does not cover the case $p=2$. It is mentioned in [28] that in the case $p=2$ one has the following inequality

$$
\left\|f-G_{m}(f, \Psi)\right\|_{L_{2}} \leq C(\Psi) \ln (m+1) \sigma_{m}(f, \Psi)_{L_{2}}
$$

We do not know if the above inequality is sharp in the sense that an extra factor $\log m$ cannot be replaced by a slower growing factor. The reader can find further discussion of this problem in [26].

We note that inequality (1.2) is known (see [28]) in the case of unconditional bases $\Psi$. It is proved in [22] that (1.2) holds for the trigonometric system $\Psi=\left\{e^{i k x}\right\}$ for all $1 \leq p \leq \infty$. It was noticed in [22] that (1.2) holds for any uniformly bounded orthonormal basis of $L_{2}$. Thus, it was known that bases satisfying very different in nature conditions - uniformly bounded orthonormal basis of $L_{2}$ or quasi-greedy basis of $L_{p}$ - both guarantee that similar Lebesgue-type inequalities (1.2) hold for greedy approximation. In this paper we continue to study Lebesgue-type inequalities for greedy approximation. We try to make a bridge between the two above conditions - uniformly bounded orthonormal basis of $L_{2}$ and quasi-greedy basis of $L_{p}$. We consider uniformly bounded quasi-greedy bases of $L_{q}$ and study Lebesgue-type inequalities in $L_{p}, q \leq p$. It turns out that even the question of existence of such bases is nontrivial. For instance, it is known (see [4]) that there is no uniformly bounded unconditional bases in $L_{p}, p \neq 2$. Quasi-greedy bases are close to unconditional bases. However, surprisingly, it turns out that there exist uniformly bounded quasi-greedy bases in all $L_{q}$ with $1<q<\infty$. We discuss this issue in Section 3 , where we present a construction of uniformly bounded quasi-greedy bases. In particular, we formulate the following theorem there.

Theorem 1.2. There exists a uniformly bounded orthonormal quasi-greedy basis $\Psi=\left\{\psi_{j}\right\}_{j=1}^{\infty}$ in $L_{p}, 1<p<\infty$, that consists of trigonometric polynomials.

We note that existence of uniformly bounded orthonormal quasi-greedy bases was proved by Nielsen [15]. The construction in [15] is a variation on a construction in [11]. The same type of construction was used in [28]. Our construction in Section 3 is a somewhat more general version of the known 
construction. We include it in the paper without proofs for the sake of completeness and because some of our results in Section 4 rely on a particular choice of the parameters in this specific construction. Conceivably, too, this more general construction may have further applications. The construction in [15] is based on the Walsh system. We should emphasize that Theorem 1.2 could also be obtained from the arguments of [15] by replacing the Walsh system by the trigonometric system.

It is known from [1] that the space $C[0,1]$ does not have quasi-greedy bases and the space $L_{1}[0,1]$ has quasi-greedy bases. In Section 4 we prove, in particular, that the space $L_{1}[0,1]$ does not have a uniformly bounded quasi-greedy Markushevich basis. This result complements a theorem of Szarek [21] on the nonexistence of a uniformly bounded Schauder basis for $L_{1}[0,1]$. On the other hand, we show that the Hardy space $H_{1}(D)$ does have a uniformly bounded quasi-greedy basis of analytic polynomials.

In Section 5 we prove Lebesgue-type inequalities for greedy approximation in $L_{p}, 2 \leq p \leq \infty$, under different assumptions on a basis $\Psi$. In that section we assume that $\Psi$ is a uniformly bounded basis. In addition we assume that $\Psi$ is a certain type basis (quasi-greedy basis, Riesz basis) in one of the spaces $L_{2}, L_{q}, 1<q<2$, or $L_{q}, 2<q<\infty$. Here is a typical result from Section 5 (see Theorem 5.2). We will often use the notation $h(p):=\left|\frac{1}{2}-\frac{1}{p}\right|$. We also use the brief notation $\|\cdot\|_{p}:=\|\cdot\|_{L_{p}}$.

Theorem 1.3. Assume that $\Psi$ is a uniformly bounded quasi-greedy basis of $L_{2}$. Then for any $m$-term polynomial

$$
t_{m}=\sum_{k \in P} b_{k} \psi_{k}, \quad|P|=m
$$

we have for $2 \leq p \leq \infty$

$$
\left\|f-G_{m}(f, \Psi)\right\|_{p} \leq\left\|f-t_{m}\right\|_{p}+C m^{h(p)} \ln (m+1)\left\|f-t_{m}\right\|_{2} .
$$

In Section 6 we continue to prove Lebesgue-type inequalities for greedy approximation in $L_{p}$ under different assumptions on a basis $\Psi$. In that section we assume that $\Psi$ is a semi-normalized quasi-greedy basis for a pair of spaces: $L_{q}, 1<q<\infty$, and $L_{p}, q \leq p$. It turns out that this assumption results in a dramatic improvement of the corresponding Lebesgue-type inequalities. It is demonstrated by the following result (see Theorem 6.1).

Theorem 1.4. Assume that $\Psi$ is a semi-normalized quasi-greedy basis for both $L_{q}$ and $L_{p}$ with $1<q \leq 2 \leq p<\infty$. Then for any $m$-term polynomial

$$
t_{m}=\sum_{k \in P} b_{k} \psi_{k}, \quad|P|=m
$$


we have

$$
\left\|f-G_{m}(f, \Psi)\right\|_{p} \leq\left\|f-t_{m}\right\|_{p}+C(p, q) \ln (m+1)\left\|f-t_{m}\right\|_{q} .
$$

We now formulate some of the Lebesgue-type inequalities obtained in the paper. We already mentioned above (see Theorem 1.1) that the Lebesguetype inequalities in $L_{p}, 1<p<\infty$, under assumption that $\Psi$ is a quasigreedy basis of $L_{p}$ were obtained in [27]. First we give a definition of a democratic basis.

Definition 1.3. We say that a basis $\Psi=\left\{\psi_{k}\right\}_{k=1}^{\infty}$ is a democratic basis for $X$ if there exists a constant $D:=D(X, \Psi)$ such that, for any two finite sets of indices $P$ and $Q$ with the same cardinality $|P|=|Q|$, we have

$$
\left\|\sum_{k \in P} \psi_{k}\right\| \leq D\left\|\sum_{k \in Q} \psi_{k}\right\|
$$

In Section 5 we prove that if $\Psi$ is both quasi-greedy and democratic then for any $f \in X$

$$
\left\|f-G_{m}(f, \Psi)\right\|_{X} \leq C \ln (m+1) \sigma_{m}(f, \Psi)_{X} .
$$

We note that it is proved in [2] that bases which are simultaneously quasigreedy and democratic are exactly almost greedy bases. As a corollary of (1.3) we obtain the Lebesgue-type inequality for a uniformly bounded quasigreedy basis of $L_{p}, 1<p<\infty$ (see Corollary 5.3):

$$
\left\|f-G_{m}(f, \Psi)\right\|_{p} \leq C(p) \ln (m+1) \sigma_{m}(f, \Psi)_{p} .
$$

Here $\sigma_{m}(f, \Psi)_{p}:=\sigma_{m}(f, \Psi)_{L_{p}}$. Comparing (1.4) with (1.2) we see that an extra assumption of uniform boundedness of the basis improves the Lebesguetype inequalities dramatically.

In Section 6, making our assumptions on the basis even stronger, we improve (1.4) to the following inequality

$$
\left\|f-G_{m}(f, \Psi)\right\|_{p} \leq C(p)(\ln (m+1))^{1 / 2} \sigma_{m}(f, \Psi)_{p},
$$

under assumption that $\Psi$ is a uniformly bounded orthonormal quasi-greedy basis of $L_{p}, 2 \leq p<\infty$.

In Section 5 we impose assumptions on the basis in the $L_{q}$ space and obtain inequalities in the $L_{p}$ space:

$$
\left\|f-G_{m}(f, \Psi)\right\|_{p} \leq C(p, q) m^{(1-q / p) / 2} \ln (m+1) \sigma_{m}(f, \Psi)_{p}
$$


under assumption that $\Psi$ is a uniformly bounded quasi-greedy basis of $L_{q}$, $1<q<\infty$, and $q \leq p \leq \infty$. We note that in the case $p=q$ inequality (1.6) turns into (1.4).

We begin a systematic presentation with Section 2, where we list some properties of quasi-greedy bases that are used in the paper.

\section{Properties of quasi-greedy bases}

\subsection{Quasi-greedy bases}

The definition of a quasi-greedy basis is given in the Introduction (see Definition 1.2). We give here an equivalent definition (see [25], p. 34). For a set of indices $\Lambda$ we define the corresponding partial sum as follows

$$
S_{\Lambda}(f):=S_{\Lambda}(f, \Psi):=\sum_{k \in \Lambda} c_{k}(f) \psi_{k} .
$$

Definition 2.1. We say that a basis $\Psi$ is quasi-greedy if there exists a constant $C_{Q}$ such that, for any $f \in X$ and any finite set of indices $\Lambda$ having the property

$$
\min _{k \in \Lambda}\left|c_{k}(f)\right| \geq \max _{k \notin \Lambda}\left|c_{k}(f)\right|
$$

we have

$$
\left\|S_{\Lambda}(f, \Psi)\right\| \leq C_{Q}\|f\|
$$

First, we present some known useful properties of quasi-greedy bases. The reader can find the following two lemmas in [25], p. 37.

Lemma 2.1. Let $\Psi$ be a quasi-greedy basis. Then, for any two finite sets of indices $A \subseteq B$ and coefficients $0<t \leq\left|c_{j}\right| \leq 1, j \in B$, we have

$$
\left\|\sum_{j \in A} c_{j} \psi_{j}\right\| \leq C(X, \Psi, t)\left\|\sum_{j \in B} c_{j} \psi_{j}\right\|
$$

It will be convenient to define the quasi-greedy constant $K$ to be the least constant such that

$$
\left\|G_{m}(f)\right\| \leq K\|f\| \quad \text { and } \quad\left\|f-G_{m}(f)\right\| \leq K\|f\|, \quad f \in X
$$

Lemma 2.2. Suppose $\Psi$ is a quasi-greedy basis with a quasi-greedy constant $K$. Then, for any real numbers $c_{j}$ and any finite set of indices $P$, we have

$$
\left(4 K^{2}\right)^{-1} \min _{j \in P}\left|c_{j}\right|\left\|\sum_{j \in P} \psi_{j}\right\| \leq\left\|\sum_{j \in P} c_{j} \psi_{j}\right\| \leq 2 K \max _{j \in P}\left|c_{j}\right|\left\|\sum_{j \in P} \psi_{j}\right\| .
$$


We present the following lemma from [1] with a proof for completeness.

Lemma 2.3. Let $\Psi$ be a quasi-greedy basis of $X$. Then for any finite set of indices $\Lambda$ we have for all $f \in X$

$$
\left\|S_{\Lambda}(f, \Psi)\right\| \leq C \ln (|\Lambda|+1)\|f\| .
$$

Proof. For a given element $f \in X$ we consider the expansion

$$
f=\sum_{k=1}^{\infty} c_{k}(f) \psi_{k} .
$$

Let a sequence $k_{j}, j=1,2, \ldots$, of the positive integers be such that

$$
\left|c_{k_{1}}(f)\right| \geq\left|c_{k_{2}}(f)\right| \geq \ldots
$$

We will use the notation

$$
a_{n}(f):=\left|c_{k_{n}}(f)\right|
$$

for the decreasing rearrangement of the coefficients of $f$. Without loss of generality assume that $f$ is normalized in such a way that guarantees that $\left|a_{1}(f)\right| \leq 1$ and consider $m:=|\Lambda| \geq 2$. Consider for integer $s \geq 0$

$$
\tau_{s}:=\left\{k: 2^{-s} \leq\left|c_{k}(f)\right|<2^{1-s}\right\} .
$$

Denote

$$
\Lambda_{s}:=\Lambda \cap \tau_{s}, \quad \Lambda^{\prime}:=\Lambda \backslash\left(\cup_{s \leq \log _{2} m} \Lambda_{s}\right) .
$$

The semi-normalization property of the basis $\Psi$ implies

$$
\left\|S_{\Lambda^{\prime}}(f)\right\| \leq \frac{2}{m}\left|\Lambda^{\prime}\right| C_{0} \leq 2 C_{0} .
$$

For $s \leq \log _{2} m$ we have

$$
S_{\Lambda_{s}}(f)=S_{\Lambda_{s}}\left(S_{\tau_{s}}(f)\right)
$$

By Lemma 2.1 we obtain

$$
\left\|S_{\Lambda_{s}}(f)\right\| \leq C\left\|S_{\tau_{s}}(f)\right\| .
$$

Our assumption that $\Psi$ is a quasi-greedy basis implies that for all $s$

$$
\left\|S_{\tau_{s}}(f)\right\| \leq C\|f\| \text {. }
$$

Thus, for all $s \leq \log _{2} m$

$$
\left\|S_{\Lambda_{s}}(f)\right\| \leq C\|f\|
$$

and, therefore,

$$
\left\|S_{\Lambda}(f)\right\| \leq C \ln (m+1)\|f\|
$$


The following Lemma 2.4 is a new result that answers Question 2 from [7]. Let

$$
f=\sum_{k=1}^{\infty} c_{k}(f) \psi_{k} .
$$

We define the following expansional best $m$-term approximation of $f$

$$
\tilde{\sigma}_{m}(f):=\tilde{\sigma}_{m}(f, \Psi):=\inf _{\Lambda,|\Lambda|=m}\left\|f-\sum_{k \in \Lambda} c_{k}(f) \psi_{k}\right\| .
$$

It is clear that

$$
\sigma_{m}(f, \Psi) \leq \tilde{\sigma}_{m}(f, \Psi)
$$

It is also clear that for an unconditional basis $\Psi$ we have

$$
\tilde{\sigma}_{m}(f, \Psi) \leq C(X, \Psi) \sigma_{m}(f, \Psi) .
$$

Lemma 2.4. Let $\Psi$ be a quasi-greedy basis of $X$. Then for all $f \in X$

$$
\tilde{\sigma}_{m}(f) \leq C \ln (m+1) \sigma_{m}(f) .
$$

Proof. For a given $\epsilon>0$ let $p_{m}$ be an $m$-term polynomial

$$
p_{m}:=\sum_{k \in P} b_{k} \psi_{k}, \quad|P|=m
$$

such that

$$
\left\|f-p_{m}\right\| \leq \sigma_{m}(f)+\epsilon .
$$

Then by Lemma 2.3 we obtain

$\tilde{\sigma}_{m}(f) \leq\left\|f-S_{P}(f)\right\|=\left\|f-p_{m}-S_{P}\left(f-p_{m}\right)\right\| \leq C \ln (m+1)\left(\sigma_{m}(f)+\epsilon\right)$.

This completes the proof of Lemma 2.4.

Remark 2.1. After our paper was submitted we were informed that Lemma 2.4 was proved independently by Garrigós, Hernández, and Oikhberg.

We now formulate a result about quasi-greedy bases in $L_{p}$ spaces. The following theorem is from [26]. We note that in the case $p=2$ Theorem 2.1 was proved in [28].

Theorem 2.1. Let $\Psi=\left\{\psi_{k}\right\}_{k=1}^{\infty}$ be a quasi-greedy basis of the $L_{p}$ space, $1<p<\infty$. Then for each $f \in X$ we have

$$
\begin{aligned}
& C_{1}(p) \sup _{n} n^{1 / p} a_{n}(f) \leq\|f\|_{p} \leq C_{2}(p) \sum_{n=1}^{\infty} n^{-1 / 2} a_{n}(f), \quad 2 \leq p<\infty ; \\
& C_{3}(p) \sup _{n} n^{1 / 2} a_{n}(f) \leq\|f\|_{p} \leq C_{4}(p) \sum_{n=1}^{\infty} n^{1 / p-1} a_{n}(f), \quad 1<p \leq 2 .
\end{aligned}
$$


Remark 2.2. Theorem 2.1 was proved in [26] under assumption that $\Psi$ is a normalized basis. That proof works for a semi-normalized basis as well.

Remark 2.3. The proof of Theorem 2.1 in [26] gives the following inequalities. Let $\Psi=\left\{\psi_{k}\right\}_{k=1}^{\infty}$ be a quasi-greedy basis of $X$. If for any set of indices $A$ of cardinality $m$ we have $\left\|\sum_{k \in A} \psi_{k}\right\|_{X} \leq C^{\prime} m^{1 / 2}$ then for each $f \in X$ we have

$$
\|f\|_{X} \leq C_{1} \sum_{n=1}^{\infty} n^{-1 / 2} a_{n}(f) .
$$

If for any set of indices $A$ of cardinality $m$ we have $\left\|\sum_{k \in A} \psi_{k}\right\|_{X} \geq c^{\prime} m^{1 / 2}$ then for each $f \in X$ we have

$$
\|f\|_{X} \geq c_{1} \sup _{n} n^{1 / 2} a_{n}(f)
$$

A general version of (2.1) was obtained in [7]. We define the fundamental function $\varphi(m):=\varphi(m, \Psi, X)$ of a basis $\Psi$ in $X$ by

$$
\varphi(m, \Psi, X):=\sup _{|A| \leq m}\left\|\sum_{k \in A} \psi_{k}\right\|
$$

Lemma 2.5. Let $\Psi$ be a quasi-greedy basis of $X$. Then for each $f \in X$ we have

$$
\|f\| \leq C \sum_{n=1}^{\infty} a_{n}(f) \varphi(n) \frac{1}{n} .
$$

Proof. It is known (see [2], p. 581) that $\varphi(n) / n$ is monotone decreasing. Therefore, by Lemma 2.2 we obtain

$$
\begin{gathered}
\|f\| \leq \sum_{s=1}^{\infty}\left\|\sum_{n=2^{s-1}}^{2^{s}-1} a_{n}(f) \psi_{k_{n}}\right\| \\
\leq C \sum_{s=1}^{\infty} a_{2^{s-1}}(f) \varphi\left(2^{s-1}\right) \leq C \sum_{n=1}^{\infty} a_{n}(f) \varphi(n) \frac{1}{n} .
\end{gathered}
$$

\subsection{Uniformly bounded quasi-greedy bases}

It is clear that any orthonormal basis of a Hilbert space $H$ is an unconditional basis and, therefore, a quasi-greedy basis of $H$. For example, the trigonometric basis is a uniformly bounded orthonormal basis of $L_{2}$. Even the question of existence of a uniformly bounded quasi-greedy basis in $L_{p}$, 
$p \neq 2$, is a nontrivial question. It is known (see [4]) that there is no uniformly bounded unconditional bases in $L_{p}, p \neq 2$. As we already mentioned in the Introduction, there are uniformly bounded quasi-greedy bases in $L_{p}$, $1<p<\infty$ [15]. We sketch a construction of such bases in Section 3. We now present some properties of these bases.

Lemma 2.6. Assume that $\Psi$ is a uniformly bounded quasi-greedy basis of $L_{q}, 1<q<\infty$. Then for any set $\Lambda$ of indices we have for $q<p \leq \infty$

$$
\left\|S_{\Lambda}(f)\right\|_{p} \leq C|\Lambda|^{(1-q / p) / 2}\left\|S_{\Lambda}(f)\right\|_{q} .
$$

We also have

$$
\left\|S_{\Lambda}(f)\right\|_{q} \leq C \ln (|\Lambda|+1)\|f\|_{q} .
$$

Proof. Inequality (2.3) follows from Lemma 2.3. We prove (2.2). We have

$$
\left\|S_{\Lambda}(f)\right\|_{\infty} \leq \sum_{k \in \Lambda}\left|c_{k}(f)\right|\left\|\psi_{k}\right\|_{\infty} \leq C \sum_{n=1}^{m} a_{n}\left(S_{\Lambda}(f)\right) .
$$

By Proposition 2.2 (see below) we continue

$$
\leq C \sum_{n=1}^{m} n^{-1 / 2}\left\|S_{\Lambda}(f)\right\|_{q} \leq C m^{1 / 2}\left\|S_{\Lambda}(f)\right\|_{q} .
$$

The above inequality combined with

$$
\|g\|_{p} \leq\|g\|_{q}^{q / p}\|g\|_{\infty}^{1-q / p}, \quad q \leq p \leq \infty
$$

gives (2.2).

We note that in the case $1<q \leq 2$ we could use Theorem 2.1 instead of Proposition 2.2.

Corollary 2.1. Assume that $\Psi$ is a uniformly bounded Riesz basis of $L_{2}$. Then for any set $\Lambda$ of indices we have for $2 \leq p \leq \infty$

$$
\left\|S_{\Lambda}(f)\right\|_{p} \leq C|\Lambda|^{h(p)}\|f\|_{2} .
$$

Proof. The case $p>2$ follows from Lemma 2.6 since Riesz bases are unconditional and hence quasi-greedy. The case $p=2$ follows from unconditionality. 


\subsection{Uniformly bounded orthonormal quasi-greedy bases}

We prove in Section 3 that there exist uniformly bounded orthonormal quasi-greedy bases in $L_{p}, 1<p<\infty$. We also prove in Section 3 that if $\Psi$ is a uniformly bounded orthonormal quasi-greedy basis in $L_{p}, 2 \leq p<\infty$ then $\Psi$ is a quasi-greedy basis of $L_{p^{\prime}}\left(p^{-1}+p^{-1}=1\right)$. Thus there are uniformly bounded bases which are quasi-greedy bases of two spaces $L_{p}$ and $L_{p^{\prime}}, 2<p<\infty$. We now present some results in this direction.

Lemma 2.7. Assume that $\Psi$ is a semi-normalized quasi-greedy basis for both $L_{q}$ and $L_{p}$ with $1<q \leq 2 \leq p<\infty$. Then for any set $\Lambda$ of indices we have

$$
\left\|S_{\Lambda}(f)\right\|_{p} \leq C \ln (|\Lambda|+1)\|f\|_{q} .
$$

Proof. Using notation $m:=|\Lambda|$ we obtain by Theorem 2.1

$$
\begin{aligned}
& \left\|S_{\Lambda}(f)\right\|_{p} \leq C_{2}(p) \sum_{n=1}^{m} n^{-1 / 2} a_{n}\left(S_{\Lambda}(f)\right) \leq C(p) \sum_{n=1}^{m} n^{-1 / 2} a_{n}(f) \\
& \leq C(p) \sum_{n=1}^{m} n^{-1 / 2} C_{3}(q)^{-1}\|f\|_{q} n^{-1 / 2} \leq C(p, q) \ln (m+1)\|f\|_{q} .
\end{aligned}
$$

This proves $(2.5)$.

Lemma 2.8. Assume that $\Psi$ is a uniformly bounded orthonormal quasigreedy basis of $L_{p}, 2<p<\infty$. Then for any set $\Lambda$ of indices we have

$$
\left\|S_{\Lambda}(f)\right\|_{2} \leq C(\ln (|\Lambda|+1))^{1 / 2}\|f\|_{p^{\prime}}
$$

and

$$
\left\|S_{\Lambda}(f)\right\|_{p} \leq C(\ln (|\Lambda|+1))^{1 / 2}\|f\|_{2} .
$$

Proof. Let $|\Lambda|=m$. By Theorem 2.2 (see below) and Theorem 2.1 we have in the case of (2.6)

$$
\left\|S_{\Lambda}(f)\right\|_{2} \leq\left(\sum_{n=1}^{m} a_{n}(f)^{2}\right)^{1 / 2} \leq C\left(\sum_{n=1}^{m} n^{-1}\|f\|_{p^{\prime}}^{2}\right)^{1 / 2} \leq C(\ln (m+1))^{1 / 2}\|f\|_{p^{\prime}} .
$$

In the case of $(2.7)$ we obtain by Theorem 2.1

$$
\begin{gathered}
\left\|S_{\Lambda}(f)\right\|_{p} \leq C \sum_{n=1}^{m} n^{-1 / 2} a_{n}(f) \\
\leq C(\ln (m+1))^{1 / 2}\left(\sum_{n=1}^{m} a_{n}(f)^{2}\right)^{1 / 2} \leq C(\ln (m+1))^{1 / 2}\|f\|_{2} .
\end{gathered}
$$


Let us discuss in more details uniformly bounded orthonormal quasigreedy bases. The existence of such bases was proved in [15]. We first recall the definition of bases which are called unconditional for constant coefficients, cf. [28].

Definition 2.2. A basis $\Psi$ is called unconditional for constant coefficients (UCC) if there exist constants $C_{1}$ and $C_{2}$ such that for each finite subset $A \subset \mathbb{N}$ and for each choice of signs $\varepsilon_{i}= \pm 1$ we have

$$
C_{1}\left\|\sum_{i \in A} \psi_{i}\right\| \leq\left\|\sum_{i \in A} \varepsilon_{i} \psi_{i}\right\| \leq C_{2}\left\|\sum_{i \in A} \psi_{i}\right\| .
$$

It is known ([28]) that quasi-greedy bases are UCC bases. To formulate our results we need some of the basic concepts of the Banach space theory from [13, Definition 1.e.12]. First, let us recall the definition of type and cotype. Let $\left\{\varepsilon_{i}\right\}$ be a sequence of independent Rademacher variables. We say that a Banach space $X$ has type $p$ if there exists a universal constant $C_{3}$ such that for $f_{k} \in X$

$$
\left(\operatorname{Ave}_{\varepsilon_{k}= \pm 1}\left\|\sum_{k=1}^{n} \varepsilon_{k} f_{k}\right\|^{p}\right)^{1 / p} \leq C_{3}\left(\sum_{k=1}^{n}\left\|f_{k}\right\|^{p}\right)^{1 / p},
$$

and $X$ is of cotype $q$ if there exists a universal constant $C_{4}$ such that for $f_{k} \in X$

$$
\left(\operatorname{Ave}_{\varepsilon_{k}= \pm 1}\left\|\sum_{k=1}^{n} \varepsilon_{k} f_{k}\right\|^{q}\right)^{1 / q} \geq C_{4}\left(\sum_{k=1}^{n}\left\|f_{k}\right\|^{q}\right)^{1 / q} .
$$

It is known that $L_{p}, 2 \leq p<\infty$ has type 2 . Consider uniformly bounded orthonormal quasi-greedy basis $\Psi=\left\{\psi_{j}\right\}_{j=1}^{\infty}$ in $L_{p}, 2<p<\infty$. Then we obtain from its orthonormality and property UCC that for any set $A$ of indices of cardinality $m$ we have

$$
\begin{aligned}
m^{1 / 2} & =\left\|\sum_{k \in A} \psi_{k}\right\|_{2} \leq\left\|\sum_{k \in A} \psi_{k}\right\|_{p} \asymp\left(\operatorname{Ave}_{\varepsilon_{k}= \pm 1}\left\|\sum_{k \in A} \varepsilon_{k} \psi_{k}\right\|_{p}^{p}\right)^{1 / p} \\
& \asymp\left\|\left(\sum_{k \in A}\left|\psi_{k}\right|^{2}\right)^{1 / 2}\right\|_{p} \leq C(p)\left(\sum_{k \in A}\left\|\psi_{k}\right\|_{p}^{2}\right)^{1 / 2} \asymp m^{1 / 2} .
\end{aligned}
$$

Equation (2.9) shows that for uniformly bounded orthonormal quasigreedy basis $\Psi=\left\{\psi_{j}\right\}_{j=1}^{\infty}$ in $L_{p}, 2<p<\infty$, we have $\varphi\left(m, \Psi, L_{p}\right) \asymp m^{1 / 2}$. In particular, (2.9) implies that $\Psi$ is democratic. We consider along with the basis $\Psi$ in $L_{p}$ its dual basis $\Psi^{*}$ in $L_{p^{\prime}}$. By orthonormality of $\Psi$ we get 
that $\Psi^{*}=\Psi$. Properties of dual bases to quasi-greedy and almost greedy bases are discussed in detail in [2]. In particular, by Proposition 4.4 and Theorem 5.4 from [2] relation $\varphi\left(m, \Psi, L_{p}\right) \asymp m^{1 / 2}$ implies that $\Psi$ is also a quasi-greedy basis of $L_{p^{\prime}}$. We formulate this conclusion as a theorem.

Theorem 2.2. Let $\Psi$ be a uniformly bounded orthonormal quasi-greedy basis $\Psi=\left\{\psi_{j}\right\}_{j=1}^{\infty}$ in $L_{p}, 2<p<\infty$. Then $\Psi$ is a quasi-greedy basis of $L_{p^{\prime}}$.

The definition of democratic basis is given in the Introduction (see Definition 1.3).

Proposition 2.1. Let $\Psi$ be a uniformly bounded quasi-greedy basis $\Psi=$ $\left\{\psi_{j}\right\}_{j=1}^{\infty}$ in $L_{q}, 1<q<\infty$. Then $\Psi$ is democratic with fundamental function $\varphi\left(m, \Psi, L_{q}\right) \asymp m^{1 / 2}$.

Proof. We give the proof only for $1<q \leq 2$ as the case $2<q<\infty$ is similar. Using the UCC property of quasi-greedy bases and using the fact that $L_{q}, 1<q \leq 2$, is of cotype 2 we obtain as in (2.9)

$$
\left\|\sum_{k \in A} \psi_{k}\right\|_{q} \asymp\left(\mathrm{Ave}_{\varepsilon_{k}= \pm 1}\left\|\sum_{k \in A} \varepsilon_{k} \psi_{k}\right\|_{q}^{2}\right)^{1 / 2} \geq C m^{1 / 2} .
$$

Also

$\left\|\sum_{k \in A} \psi_{k}\right\|_{q} \asymp\left(\mathrm{Ave}_{\varepsilon_{k}= \pm 1}\left\|\sum_{k \in A} \varepsilon_{k} \psi_{k}\right\|_{q}^{2}\right)^{1 / 2} \leq\left(\mathrm{Ave}_{\varepsilon_{k}= \pm 1}\left\|\sum_{k \in A} \varepsilon_{k} \psi_{k}\right\|_{2}^{2}\right)^{1 / 2} \leq C m^{1 / 2}$.

Combination of Proposition 2.1 and Remark 2.3 gives the following inequalities which we will often use.

Proposition 2.2. Let $\Psi$ be a uniformly bounded quasi-greedy basis $\Psi=$ $\left\{\psi_{j}\right\}_{j=1}^{\infty}$ in $L_{q}, 1<q<\infty$. Then we have for $f \in L_{q}$

$$
c_{1}(q) \sup _{n} n^{1 / 2} a_{n}(f) \leq\|f\|_{q} \leq C_{1}(q) \sum_{n=1}^{\infty} n^{-1 / 2} a_{n}(f) .
$$

This proposition implies the following analog of Lemma 2.7.

Lemma 2.9. Assume that $\Psi$ is a uniformly bounded quasi-greedy basis $\Psi=$ $\left\{\psi_{j}\right\}_{j=1}^{\infty}$ in $L_{q}$ and $L_{p}, 1<q, p<\infty$. Then for any set $\Lambda$ of indices we have

$$
\left\|S_{\Lambda}(f)\right\|_{p} \leq C \ln (|\Lambda|+1)\|f\|_{q}
$$


Proof. Let $|\Lambda|=m$. By Proposition 2.2 we obtain

$$
\begin{gathered}
\left\|S_{\Lambda}(f)\right\|_{p} \leq C_{1}(p) \sum_{n=1}^{m} n^{-1 / 2} a_{n}(f) \\
\leq c_{1}(q)^{-1} C_{1}(p) \sum_{n=1}^{m} n^{-1}\|f\|_{q} \leq C \ln (|\Lambda|+1)\|f\|_{q} .
\end{gathered}
$$

\section{Construction of quasi-greedy bases}

In this section we describe without proofs a general scheme of construction of a quasi-greedy basis out of a given basis with special properties. This scheme generalizes the one used by Wojtaszczyk in [28]. Both schemes are based on the orthogonal Haar-type matrices, used firstly by Olevskii to construct orthogonal systems (see [5], p. 120, [16], [17]). For the proofs of these results we refer the reader to [3]

\subsection{Assumptions}

Let $X$ be a separable Banach space and $\Phi=\left\{\varphi_{j}\right\}_{j=1}^{\infty}$ be a semi-normalized basis of $X, 0<c_{0} \leq\left\|\varphi_{j}\right\| \leq C_{0}$. We assume that $\Phi$ is a Besselian basis of $X$ : for any

$$
f=\sum_{j=1}^{\infty} c_{j}(f) \varphi_{j}
$$

we have

$$
\left(\sum_{j=1}^{\infty}\left|c_{j}(f)\right|^{2}\right)^{1 / 2} \leq C_{1}\|f\| .
$$

Assume that $\Phi$ can be split into two systems $F=\left\{f_{s}\right\}_{s=1}^{\infty}, f_{s}=\varphi_{m(s)}$ and $E=\left\{e_{j}\right\}_{j=1}^{\infty}, e_{j}=\varphi_{n(j)}$ with increasing sequences $\{m(s)\}$ and $\{n(j)\}$ in such a way that $E$ has the following special property. For any sequence $\left\{c_{j}\right\}$ we have

$$
\left\|\sum_{j=1}^{\infty} c_{j} e_{j}\right\| \leq C_{2}\left(\sum_{j=1}^{\infty}\left|c_{j}\right|^{2}\right)^{1 / 2} .
$$

In our construction of quasi-greedy bases we will use special matrices. Let a collection of matrices $\mathcal{A}=\{A(n)\}_{n=1}^{\infty}, A(n)$ is of size $n \times n$, satisfy the following properties. 
M1. Singular numbers of matrices $A(n)$ and their inverse $A(n)^{-1}$ are uniformly bounded:

$$
s_{j}(A(n)) \leq C_{3} ; \quad s_{j}\left(A(n)^{-1}\right) \leq C_{3} .
$$

M2. For the elements of the first column of matrix $A(n)=\left[a_{i j}(n)\right]$ we have

$$
\left|a_{i 1}(n)\right| \leq C_{4} n^{-1 / 2}
$$

\subsection{Construction}

Let $\left\{n_{k}\right\}_{k=0}^{\infty}, n_{0}=0$, be an increasing sequence of integers such that

$$
n_{k+1} \geq n_{k}^{2}
$$

For a fixed natural number $k$ we pick the basis elements

$$
g_{1}^{k}:=f_{k}, \quad g_{i}^{k}:=e_{S_{k-1}+i-1}, \quad i=2, \ldots, n_{k},
$$

where $\left\{S_{j}\right\}$ is defined recursively as

$$
S_{j}=S_{j-1}+n_{j}-1, \quad j=1,2, \ldots, \quad S_{0}=0 .
$$

We build a new system of elements $\left\{\psi_{i}^{k}\right\}_{i=1}^{n_{k}}$ using a matrix $A\left(n_{k}\right)$ in the following way:

$$
\left(\psi_{1}^{k}, \ldots, \psi_{n_{k}}^{k}\right)^{T}=A\left(n_{k}\right)\left(g_{1}^{k}, \ldots, g_{n_{k}}^{k}\right)^{T}
$$

In other words, for $i \in\left[1, n_{k}\right]$ we have

$$
\psi_{i}^{k}=\sum_{j=1}^{n_{k}} a_{i j}\left(n_{k}\right) g_{j}^{k} .
$$

We begin with a property of the system $G:=\left\{g_{i}^{k}\right\}_{i=1, k=1}^{n_{k}, \infty}=\left\{g_{j(k, i)}\right\}$ ordered in the lexicographical way: $j\left(k^{\prime}, i^{\prime}\right)>j(k, i)$ if either $k^{\prime}>k$ or $k^{\prime}=k$ and $i^{\prime}>i$.

Proposition 3.1. The system $G$ is a Besselian basis of $X$.

We define the system $\Psi=\left\{\psi_{i}^{k}\right\}_{i=1, k=1}^{n_{k}, \infty}=\left\{\psi_{j(k, i)}\right\}$ ordered in the lexicographical way: $j\left(k^{\prime}, i^{\prime}\right)>j(k, i)$ if either $k^{\prime}>k$ or $k^{\prime}=k$ and $i^{\prime}>i$. The following theorem summarizes the properties of $\Psi$. M1 we obtain

Theorem 3.1. The basis $\Psi$ is a Besselian quasi-greedy basis of $X$. 


\subsection{Extra assumptions}

First of all we note that if $\Phi \subset H$ is from a Hilbert space $H$ and forms an orthonormal basis there then $G$ also forms an orthonormal basis in $H$. Second, if matrices $A(n)$ are orthogonal matrices then $\Psi$ is an orthonormal basis of $H$.

Next, assume that $Y$ is a subspace of $X$ with a stronger norm: $\|f\|_{X} \leq$ $\|f\|_{Y}$. Assume that the basis $\Phi$ is from $Y$ and $\left\|\varphi_{j}\right\|_{Y} \leq B, j=1, \ldots$ We impose an extra assumption on the matrices $A(n)$ too.

M3. Assume that for all $n$

$$
\sum_{j=1}^{n}\left|a_{i j}(n)\right| \leq C_{5} .
$$

Under condition M3, which is satisfied by the Haar matrices, we easily derive from the definition of $\Psi$ that

$$
\left\|\psi_{i}^{k}\right\|_{Y} \leq C_{5} B
$$

Let $X=L_{p}(0,2 \pi), 2<p<\infty, Y=L_{\infty}(0,2 \pi)$. Consider $\Phi=\mathcal{T}$ to be the trigonometric system $\left\{e^{i k x}\right\}$. Define $E:=\left\{e^{i 2^{j} x}\right\}_{j=1}^{\infty}$. It is well known that (3.3) holds for this system. By Riesz theorem $\mathcal{T}$ is a basis of $L_{p}$, $1<p<\infty$. Trivially, $\mathcal{T}$ has Besselian property in $L_{p}, 2<p<\infty$. Thus, applying the above construction for the case where the matrices $A(n)$ are orthogonal we obtain the following theorem.

Theorem 3.2. There exists a uniformly bounded orthonormal system $\Psi=$ $\left\{\psi_{j}\right\}_{j=1}^{\infty}$ consisting of trigonometric polynomials which is a quasi-greedy basis for $L_{p}[0,1]$ for all $1<p<\infty$.

Proof. The quasi-greedy property holds for all $p>2$ by the preceding discussion. It holds also for $1<p<2$ by the duality result Theorem 2.2.

Remark 3.1. The orthonormal system constructed in Theorem 3.2 will also be a quasi-greedy basis for other function spaces, including the Lorentz spaces $L_{p, q}[0,1]$ for $1<p<\infty, 1 \leq q<\infty$.

Remark 3.2. Theorem 3.2 can also be obtained from the construction of [15] by replacing the Walsh system by the trigonometric system. We thank the referee for this observation. 


\section{Uniformly bounded quasi-greedy systems}

The main result of this section is that there is no analog of Theorem 3.2 for $L_{1}[0,1]$. It is known that $L_{1}[0,1]$ has a quasi-greedy basis [1, Theorem 7.1] and, by a theorem of Szarek [21], that $L_{1}[0,1]$ does not admit any uniformly integrable Schauder basis (see also [8]). On the other hand, the trigonometric system is a uniformly bounded Markushevich basis. Therefore, it is natural to ask whether $L_{1}[0,1]$ admits a uniformly bounded (or uniformly integrable) quasi-greedy Markushevich basis $\Psi$. We answer this question negatively.

First, we recall the relevant definitions. Let $X$ be a separable Banach space. Let $\Psi=\left\{\psi_{j}\right\}_{j=1}^{\infty} \subset X$ be a semi-normalized fundamental system, i.e. there exist positive constants $a$ and $b$ such that

$$
a \leq\left\|\psi_{j}\right\| \leq b \quad(j \geq 1)
$$

with biorthogonal sequence $\left\{\psi_{j}^{*}\right\}_{j=1}^{\infty} \subset X^{*}$. $\Psi$ is said to be a Markushevich basis if the mapping $f \mapsto\left\{\psi_{j}^{*}(f)\right\}_{j=1}^{\infty}(f \in X)$ is one-one. In other words, each $f \in X$ is uniquely determined by its coefficient sequence $\left\{\psi_{j}^{*}(f)\right\}_{j=1}^{\infty}$. We say that $\Psi$ is quasi-greedy if there exists a constant $C$ such that

$$
\left\|G_{m}(f, \Psi)\right\| \leq C\|f\| \quad(m \geq 1, f \in X) .
$$

Wojtaszczyk [28] proved that (4.2) is equivalent to the norm convergence of $\left\{G_{m}(f)\right\}$ to $f$ for all $f \in X$.

It follows easily from (4.1) and (4.2) that $\left\{\psi_{j}^{*}\right\}_{j=1}^{\infty}$ is semi-normalized in $X^{*}$. Indeed, for $f \in X$, we have

$$
\left|\psi_{j}^{*}(f)\right| \leq a_{1}(f) \leq(1 / a)\left\|G_{1}(f)\right\| \leq(C / a)\|f\|,
$$

and hence $\left\|\psi_{j}^{*}\right\| \leq C / a$. On the other hand, since $\psi_{j}^{*}\left(\psi_{j}\right)=1$, we also have $\left\|\psi_{j}^{*}\right\| \geq 1 /\left\|\psi_{j}\right\| \geq 1 / b$.

The following result was proved for quasi-greedy bases (actually for the larger class of thresholding-bounded bases) in [1, Lemma 8.2]. The proof easily carries over to quasi-greedy Markushevich bases (cf. also the proof of Lemma 2.3 above).

Proposition 4.1. Suppose that $\Psi$ is a semi-normalized quasi-greedy Markushevich basis for $X$. There exists a constant $C$ such that for all finite sets $\Lambda \subset \mathbb{N}$ with $|\Lambda|=N \geq 2$, we have

$$
\max _{ \pm}\left\|\sum_{n \in \Lambda} \pm \psi_{n}^{*}(f) \psi_{n}\right\| \leq C \ln N\|f\| \quad(f \in X)
$$


In particular,

$$
\left\|S_{\Lambda}(f)\right\| \leq C \ln N\|f\| \quad(f \in X) .
$$

Recall that a bounded operator $T: X \rightarrow Y$, where $X$ and $Y$ are Banach spaces, is absolutely summing if there exists a constant $C$ such that, for all $n \geq 1$ and for all finite sequences $\left\{f_{j}\right\}_{j=1}^{n} \subset X$, we have

$$
\sum_{j=1}^{n}\left\|T\left(f_{j}\right)\right\| \leq C \max _{ \pm}\left\|\sum_{j=1}^{n} \pm f_{j}\right\| .
$$

The smallest such constant is denoted $\pi_{1}(T)$. A Banach space $X$ is called a GT space [19] if every bounded operator $T: X \rightarrow \ell_{2}$ is absolutely summing. $X$ is a GT space if and only if there exists a constant $B$ such that $\pi_{1}(T) \leq$ $B\|T\|$ for all bounded $T: X \rightarrow \ell_{2}$. Grothendieck [6] proved that $L_{1}(\mu)$ spaces are GT spaces.

The proof of the following result is based on the methods used in $[1$, Section 8].

Theorem 4.1. Suppose that $X$ is a GT space. Let $\Psi$ be a semi-normalized quasi-greedy Markushevich basis for $X$. Then $\Psi$ is democratic and its fundamental function satisfies $\varphi(n) \asymp n$.

For $1 \leq p \leq \infty$, recall that a Markushevich basis $\Psi$ is said to be $p$ Besselian if there exists a constant $C_{p}$ such that

$$
\left(\sum_{n=1}^{\infty}\left|\psi_{n}^{*}(f)\right|^{p}\right)^{1 / p} \leq C_{p}\|f\| \quad(f \in X)
$$

(with the obvious modification for $p=\infty$ ). Since $\psi$ is quasi-greedy, $C_{\infty}=$ $\sup _{n \geq 1}\left\|\psi_{n}^{*}\right\|<\infty$, so $\Psi$ is $\infty$-Besselian.

We will derive Theorem 4.1 from the following Theorem 4.2.

Theorem 4.2. Suppose that $\Psi$ is a semi-normalized quasi-greedy Markushevich basis for a GT space $X$. Then $\Psi$ is $r$-Besselian for all $r>1$.

We need the following key lemma.

Lemma 4.1. Suppose that $\Psi$ is a semi-normalized quasi-greedy Markushevich basis for a GT space $X$. If $\Psi$ is $p$-Besselian for some $2 \leq p \leq \infty$, then $\Psi$ is $r$-Besselian for all $r$ satisfying $1 / r<1 / p+1 / 2$.

Proof. We shall give the proof for the case $2<p<\infty$ as the case $p=\infty$ requires only minor changes. Let $1 / s=1 / p+1 / 2$. Suppose that $\Lambda \subset \mathbb{N}$, 
with $|\Lambda|=N$, and that $\left(\eta_{n}\right)_{n \in \Lambda}$ is any fixed choice of signs. Choose $f \in X$, with $\|f\|=1$, such that

$$
\sum_{n \in \Lambda} \eta_{n} \psi_{n}^{*}(f) \geq \frac{1}{2}\left\|\sum_{n \in \Lambda} \eta_{n} \psi_{n}^{*}\right\|
$$

Next consider $T: X \rightarrow \ell_{2}(\Lambda)$ defined as follows:

$$
T(g)=\left(\psi_{n}^{*}(g)\left|\psi_{n}^{*}(f)\right|^{s-1}\right)_{n \in \Lambda} \quad(g \in X) .
$$

Then, applying Hölder's inequality and using the fact that $\Psi$ is $p$-Besselian, we get

$$
\begin{aligned}
\|T(g)\| & =\left(\sum_{n \in \Lambda}\left|\psi_{n}^{*}(f)\right|^{2 s-2}\left|\psi_{n}^{*}(g)\right|^{2}\right)^{1 / 2} \\
& \leq\left(\sum_{n \in \Lambda}\left|\psi_{n}^{*}(f)\right|^{s}\right)^{1-1 / s}\left(\sum_{n \in \Lambda}\left|\psi_{n}^{*}(g)\right|^{p}\right)^{1 / p} \\
& \leq C_{p}\left(\sum_{n \in \Lambda}\left|\psi_{n}^{*}(f)\right|^{s}\right)^{1-1 / s}\|g\| .
\end{aligned}
$$

Hence $\|T\| \leq C_{p}\left(\sum_{n \in \Lambda}\left|\psi_{n}^{*}(f)\right|^{s}\right)^{1-1 / s}$. Since $X$ is a GT space, we have

$$
\begin{aligned}
\sum_{n \in \Lambda}\left|\psi_{n}^{*}(f)\right|^{s} & =\sum_{n \in \Lambda}\left|\psi_{n}^{*}(f)\right|\left\|T\left(\psi_{n}\right)\right\| \\
& \leq B\|T\| \sup _{\varepsilon_{n}= \pm 1}\left\|\sum_{n \in \Lambda} \varepsilon_{n} \psi_{n}^{*}(f) \psi_{n}\right\| \\
& \leq B C_{p}\left(\sum_{n \in \Lambda}\left|\psi_{n}^{*}(f)\right|^{s}\right)^{1-1 / s} \sup _{\varepsilon_{n}= \pm 1}\left\|\sum_{n \in \Lambda} \varepsilon_{n} \psi_{n}^{*}(f) \psi_{n}\right\|
\end{aligned}
$$

Thus,

$$
\left(\sum_{n \in \Lambda}\left|\psi_{n}^{*}(f)\right|^{s}\right)^{1 / s} \leq B C_{p} \sup _{\varepsilon_{n}= \pm 1}\left\|\sum_{n \in \Lambda} \varepsilon_{n} \psi_{n}^{*}(f) \psi_{n}\right\| .
$$

Since $|\Lambda|=N$, Proposition 4.1 yields

$$
\sup _{\varepsilon_{n}= \pm 1}\left\|\sum_{n \in \Lambda} \varepsilon_{n} \psi_{n}^{*}(f) \psi_{n}\right\| \leq C^{\prime}(\ln N)
$$

where $C^{\prime}$ is independent of $N$. Hence

$$
\left(\sum_{n \in \Lambda}\left|\psi_{n}^{*}(f)\right|^{s}\right)^{1 / s} \leq B C^{\prime} C_{p}(\ln N) .
$$

Thus,

$$
\begin{aligned}
\left\|\sum_{n \in \Lambda} \eta_{n} \psi_{n}^{*}\right\| & \leq 2 \sum_{n \in \Lambda} \eta_{n} \psi_{n}^{*}(f) \\
& \leq 2\left(\sum_{n \in \Lambda}\left|\psi_{n}^{*}(f)\right|^{s}\right)^{1 / s} N^{1-1 / s} \\
& \leq B C^{\prime} C_{p}(\ln N) N^{1-1 / s}
\end{aligned}
$$


Now suppose that $g \in X$ with $\|g\|=1$. For $a>0$, let

$$
\Lambda(a)=\left\{n \in \mathbb{N}:\left|\psi_{n}^{*}(g)\right| \geq a\right\} \quad \text { and } \quad N(a)=|\Lambda(a)|
$$

Then, for some choice of signs $\left(\eta_{n}\right)$, we have

$$
\begin{aligned}
a N(a) & \leq \sum_{n \in \Lambda(a)} \eta_{n} \psi_{n}^{*}(g) \\
& \leq\left\|\sum_{n \in \Lambda(a)} \eta_{n} \psi_{n}^{*}\right\| \\
& \leq B C^{\prime} C_{p}(\ln N(a)) N(a)^{1-1 / s}
\end{aligned}
$$

Thus, for some constant $C^{\prime \prime}$, we have $N(a) \leq C^{\prime \prime} a^{-t}$ provided $t$ satisfies

$$
\frac{1}{r}<\frac{1}{t}<\frac{1}{s}
$$

Note that

$$
\sup _{n \geq 1}\left|\psi_{n}^{*}(g)\right| \leq \sup _{n \geq 1}\left\|\psi_{n}^{*}\right\|_{\infty}=C_{\infty}
$$

Hence

$$
\begin{aligned}
\sum_{n=1}^{\infty}\left|\psi_{n}^{*}(g)\right|^{r} & \leq \sum_{n=0}^{\infty} N\left(2^{-n} C_{\infty}\right)\left(2^{1-n} C_{\infty}\right)^{r} \\
& \leq 2^{r} C^{\prime \prime} \sum_{n=0}^{\infty}\left(2^{-n} C_{\infty}\right)^{r-t}<\infty
\end{aligned}
$$

Hence $\Psi$ is $r$-Besselian.

Applying the lemma twice, starting with $p=\infty$, it follows that $\Psi$ is $r$-Besselian for all $r>1$, which proves Theorem 4.2.

Proof of Theorem 4.1. By Theorem 4.2, $\Psi$ is 2-Besselian with constant $C_{2}<$ $\infty$. Hence, for every finite $\Lambda \subset \mathbb{N}$, the mapping $T: X \rightarrow \ell_{2}(\Lambda)$ given by $f \mapsto\left(\psi_{n}^{*}(f)\right)_{n \in \Lambda}$ satisfies $\|T\| \leq C_{2}$. Since $X$ is a GT space the absolutely summing norm of $T$ satisfies $\pi_{1}(T) \leq B C$. Thus,

$$
|\Lambda|=\sum_{n \in \Lambda}\left\|T\left(\psi_{n}\right)\right\|_{2} \leq B C \max _{ \pm}\left\|\sum_{n \in \Lambda} \pm \psi_{n}\right\|
$$

Since $\Psi$ is quasi-greedy, and hence unconditional for constant coefficients, it follows that $\varphi(n) \asymp n$.

From [2, Lemma 3.2] and Theorem 4.1 we obtain the following result. 
Corollary 4.1. Suppose that $\Psi$ is a semi-normalized quasi-greedy Markushevitch basis for a GT space $X$. There exists a constant $C$ such that for all $g \in X$ we have

$$
a_{n}(g) \leq C n^{-1}\|g\|
$$

Recall that a system $\left\{f_{j}\right\} \subset L_{1}[0,1]$ is uniformly integrable if, given $\varepsilon>0$, there exists $\delta>0$ such that if $\lambda(A)<\delta$, where $\lambda$ denotes Lebesgue measure, then $\int_{A}\left|f_{j}\right| d \lambda<\varepsilon$ for all $j \geq 1$. Clearly, uniformly bounded systems are uniformly integrable.

Theorem 4.3. Let $\Psi$ be a semi-normalized quasi-greedy Markushevich basis for $L_{1}[0,1]$. Then no subsequence of $\Psi$ is uniformly integrable. Hence every subsequence of $\Psi$ contains a further subsequence equivalent to the unit vector basis of $\ell_{1}$.

Proof. Let $\left\{f_{j}\right\} \subset L_{1}[0,1]$ be any uniformly integrable system. Given $\varepsilon>0$, choose $M>0$ such that $\left\|f_{j} \chi_{\left\{\left|f_{j}\right|>M\right\}}\right\|_{1}<\varepsilon$ for all $j$. Then

$$
\mathrm{Ave}_{ \pm}\left\|\sum_{j=1}^{n} \pm f_{j}\right\|_{1} \leq n \varepsilon+\mathrm{Ave}_{ \pm}\left\|\sum_{j=1}^{n} \pm f_{j} \chi_{\left\{\left|f_{j}\right| \leq M\right\}}\right\|_{2} \leq n \varepsilon+M \sqrt{n}
$$

Hence $\mathrm{Ave}_{ \pm}\left\|\sum_{j=1}^{n} \pm f_{j}\right\|_{1}=o(n)$. Since $L_{1}[0,1]$ is a GT space, Theorem 4.1 implies that $\left\{f_{j}\right\}$ is not a subsequence of any quasi-greedy Markushevich basis. Finally, it is well-known that semi-normalized sequences in $L_{1}[0,1]$ are either uniformly integrable or contain a subsequence equivalent to the unit vector basis of $\ell_{1}$.

Remark 4.1. Complemented subspaces of $L_{1}$ spaces are GT spaces. Hence the previous theorem extends to quasi-greedy Markushevich bases of complemented (infinite-dimensional) subspaces of $L_{1}[0,1]$. A related result of Popov [20] asserts that complemented subspaces of $L_{1}[0,1]$ do not admit a uniformly integrable Schauder basis.

Next we consider the Hardy spaces $H_{p}(D)(1 \leq p<\infty)$ of analytic functions on the disk $D:=\{z \in \mathbb{C}:|z|<1\}$ equipped with the norm

$$
\|f\|_{p}=\sup _{0<r<1}\left(\frac{1}{2 \pi} \int_{0}^{2 \pi} \mid f\left(\left.r e^{i \theta}\right|^{p} d \theta\right)^{1 / p} .\right.
$$

Using the system $\left\{z^{n}\right\}_{n=0}^{\infty}$ instead of $\mathcal{T}$ in the proof of Theorem 3.2 yields the following result.

Theorem 4.4. There exists an orthonormal system of uniformly bounded analytic polynomials which is a quasi-greedy basis for $H_{p}(D)$ for $1<p<\infty$. 
Using some deep results from Banach space theory we can extend the latter result also to the case $p=1$.

Theorem 4.5. $H_{1}(D)$ admits a semi-normalized uniformly bounded quasigreedy basis of analytic polynomials.

Proof. By Paley's inequality [18]

$$
\left(\sum_{n=1}^{\infty}\left|\hat{f}\left(2^{n}\right)\right|^{2}\right)^{1 / 2} \asymp\left\|\sum_{n=1}^{\infty} \hat{f}\left(2^{n}\right) z^{2^{n}}\right\|_{1} \leq 2\|f\|_{1}
$$

Hence $P(f)=\sum_{n=1}^{\infty} \hat{f}\left(2^{n}\right) z^{2^{n}}$ is a bounded projection on $H_{1}(D)$. Let $X:=$ ker $P$ and let $H:=\left[e_{j}\right]_{j=1}^{\infty}$, where $e_{j}:=z^{2^{j}}$. Then $H_{1}(D)$ is linearly isomorphic to $X \oplus H$ (equipped with the sum norm), which in turn is isomorphic to $X \oplus \ell_{2}$. Since $X$ contains a complemented subspace isomorphic to $\ell_{2}$ (e.g., the subspace spanned by $\left.\left(z^{3^{j}}\right)_{j=1}^{\infty}\right)$, it follows that $X$ is also isomorphic to $X \oplus \ell_{2}$ and thus also isomorphic to $H_{1}(D)$. Hence, by a theorem of Maurey [14], $X$ has a normalized unconditional basis $\left(f_{j}\right)_{j=1}^{\infty}$. The intersection of $X$ with the linear space of analytic polynomials is dense in $X$. Hence we may assume that each $f_{j}$ is an analytic polynomial. Since $H_{1}(D)$ has cotype 2 (see (2.8)), it follows that $\left\{f_{j}\right\}_{j=1}^{\infty}$ is Besselian. Then $\Phi=\left\{f_{j}\right\}_{j=1}^{\infty} \cup\left\{e_{j}\right\}_{j=1}^{\infty}$ is a basis for $H_{1}(D)$ satisfying (3.2) and (3.3). Assume the matrices $\{A(n)\}$ satisfy M1-M3 and, in addition, that $n_{k} \geq\left\|f_{k}\right\|_{\infty}^{2}$. By the construction, the system $\Psi$ is a semi-normalized quasi-greedy basis. Moreover, by M2 and M3,

$$
\sup _{j \geq 1}\left\|\psi_{j}\right\|_{\infty} \leq \sup _{k \geq 1} \frac{\left\|f_{k}\right\|_{\infty}}{\sqrt{n_{k}}}+C_{5} \sup _{k \geq 1}\left\|e_{k}\right\| \leq 1+C_{5} .
$$

Thus, $\Psi$ is uniformly bounded.

Finally, let us mention that Theorem 3.2 may be generalized to certain closed subspaces of $L_{p}[0,1]$, for $p>2$, including those spanned by any subsequence of the trigonometric system. Recall that $\left\{\psi_{j}\right\} \subset L_{2}[0,1]$ is a Riesz sequence if $\left\|\sum c_{j} \psi_{j}\right\|_{2} \asymp\left(\sum\left|c_{j}\right|^{2}\right)^{1 / 2}$ for all scalars $\left\{c_{j}\right\}$.

Proposition 4.2. Let $X$ be a closed subspace of $L_{p}[0,1]$ for $2 \leq p<\infty$. Suppose that $X$ has a uniformly bounded Schauder basis $\left\{\psi_{j}\right\}$ which is a Riesz sequence in $L_{2}[0,1]$. Then $X$ admits a uniformly bounded quasi-greedy basis.

Proof. Since $L_{p}[0,1]$ has an unconditional basis and $\left\{\psi_{j}\right\}$ is weakly null, it follows by a standard "gliding hump" argument that some subsequence 
$\left\{\psi_{j}\right\}_{j \in A}$ is unconditional. Since $L_{p}[0,1]$ has type 2 (for the upper estimate) and $\left\{\psi_{j}\right\}$ is a Riesz sequence in $L_{2}[0,1]$ (for the lower estimate) it follows that $\left\|\sum_{j \in A} c_{j} \psi_{j}\right\|_{p} \asymp\left(\sum_{j \in A}\left|c_{j}\right|^{2}\right)^{1 / 2}$ for all scalars $\left\{c_{j}\right\}$, i.e., $\left\{\psi_{j}\right\}_{j \in A}$ is a sequence in $X$ that is equivalent to the unit vector basis of $\ell_{2}$. Since $\left\{\psi_{j}\right\}$ is a Riesz sequence in $L_{2}[0,1]$, we have, for all $f \in X$,

$$
\left\|\sum c_{j}(f) \psi_{j}\right\|_{p} \geq\left\|\sum c_{j}(f) \psi_{j}\right\|_{2} \geq k_{1}\left(\sum_{j \in A}\left|c_{j}(f)\right|^{2}\right)^{1 / 2} \geq k_{2}\left\|\sum_{j \in A} c_{j}(f) \psi_{j}\right\|_{p}
$$

where $k_{1}$ and $k_{2}$ are constants. Hence the projection $P f=\sum_{j \in A} c_{j}(f) \psi_{j}$ is bounded on $X$, which implies that $X$ is linearly isomorphic to $\left[\psi_{j}\right]_{j \notin A} \oplus$ $\left[\psi_{j}\right]_{j \in A}$. The fact that $\left\{\psi_{j}\right\}$ is a Riesz sequence in $L_{2}[0,1]$ implies that $\left\{\psi_{j}\right\}$ is a (uniformly bounded) Besselian basis for $X$. The proof is completed as in the discussion preceding Theorem 3.2.

\section{Lebesgue-type inequalities I}

Our main interest in this section is to prove Lebesgue-type inequalities for greedy approximation in $L_{p}, 2 \leq p \leq \infty$, under different assumptions on a basis $\Psi$. In this section we assume that $\Psi$ is a uniformly bounded basis. In addition we assume that $\Psi$ is a certain type basis (quasi-greedy basis, Riesz basis) in one of the spaces $L_{2}, L_{q}, 1<q<2$, or $L_{q}, 2<q<\infty$. We will often use the following lemma.

Lemma 5.1. Suppose that $X \subset Y$ are two Banach spaces such that $\|\cdot\|_{Y} \leq$ $\|\cdot\|_{X}$. Assume that a basis of $X \Psi$ satisfies the following property: For any set of indices $\Lambda$

$$
\left\|S_{\Lambda}(f)\right\|_{X} \leq w(|\Lambda|)\|f\|_{Y}
$$

Then for each $f \in X$ and any $m$-term polynomial

$$
p_{m}=\sum_{k \in P} b_{k} \psi_{k}, \quad|P|=m
$$

we have

$$
\left\|f-S_{P}(f)\right\|_{X} \leq\left\|f-p_{m}\right\|_{X}+w(m)\left\|f-p_{m}\right\|_{Y}
$$

Proof. It is a simple one line proof. We have

$\left\|f-S_{P}(f)\right\|_{X}=\| f-p_{m}(f)-S_{P}\left(f-p_{m}(f)\left\|_{X} \leq\right\| f-p_{m}\left\|_{X}+w(m)\right\| f-p_{m} \|_{Y}\right.$ 
We now proceed to a systematic presentation of new results.

Theorem 5.1. Assume that $\Psi$ is a uniformly bounded Riesz basis of $L_{2}$. Then for any $m$-term polynomial

$$
t_{m}=\sum_{k \in P} b_{k} \psi_{k}, \quad|P|=m
$$

we have for $2 \leq p \leq \infty$

$$
\left\|f-G_{m}(f, \Psi)\right\|_{p} \leq\left\|f-t_{m}\right\|_{p}+C m^{h(p)}\left\|f-t_{m}\right\|_{2} .
$$

Corollary 5.1. Assume that $\Psi$ is a uniformly bounded Riesz basis of $L_{2}$. Then we have for $2 \leq p \leq \infty$

$$
\left\|f-G_{m}(f, \Psi)\right\|_{p} \leq C m^{h(p)} \sigma_{m}(f, \Psi)_{p} .
$$

Proof. Denote by $Q$ the set of indices picked by the greedy algorithm after $m$ iterations

$$
G_{m}(f):=G_{m}(f, \Psi)=\sum_{k \in Q} c_{k}(f) \psi_{k} .
$$

We use the representation

$$
f-G_{m}(f)=f-S_{Q}(f)=f-S_{P}(f)+S_{P}(f)-S_{Q}(f) .
$$

First, we bound $\left\|f-S_{P}(f)\right\|_{p}$. By Lemma 5.1 and Lemma 2.1 we get

$$
\left\|f-S_{P}(f)\right\|_{p} \leq\left\|f-t_{m}\right\|_{p}+C m^{h(p)}\left\|f-t_{m}\right\|_{2} .
$$

Second, we write

$$
\begin{gathered}
\left\|S_{P}(f)-S_{Q}(f)\right\|_{p}=\left\|S_{P \backslash Q}(f)-S_{Q \backslash P}(f)\right\|_{p} \\
\leq\left\|S_{P \backslash Q}(f)\right\|_{p}+\left\|S_{Q \backslash P}(f)\right\|_{p} .
\end{gathered}
$$

Using Lemma 2.1 we obtain

$$
\left\|S_{P}(f)-S_{Q}(f)\right\|_{p} \leq C m^{h(p)}\left(\left\|S_{P \backslash Q}(f)\right\|_{2}+\left\|S_{Q \backslash P}(f)\right\|_{2}\right) .
$$

The definition of $Q$ implies

$$
\begin{gathered}
\left\|S_{P \backslash Q}(f)\right\|_{2} \leq C\left(\sum_{k \in P \backslash Q}\left|c_{k}(f)\right|^{2}\right)^{1 / 2} \\
\leq C\left(\sum_{k \in Q \backslash P}\left|c_{k}(f)\right|^{2}\right)^{1 / 2} \leq C\left\|S_{Q \backslash P}(f)\right\|_{2} .
\end{gathered}
$$

Next,

$$
\left\|S_{Q \backslash P}(f)\right\|_{2}=\left\|S_{Q \backslash P}\left(f-t_{m}\right)\right\|_{2} \leq C\left\|f-t_{m}\right\|_{2} .
$$

Combining (5.1) - (5.6) we complete the proof of Theorem 5.1. 
We now impose a little weaker assumption on a basis $\Psi$ than the one in Theorem 5.1.

Theorem 5.2. Assume that $\Psi$ is a uniformly bounded quasi-greedy basis of $L_{2}$. Then for any $m$-term polynomial

$$
t_{m}=\sum_{k \in P} b_{k} \psi_{k}, \quad|P|=m
$$

we have for $2 \leq p \leq \infty$

$$
\left\|f-G_{m}(f, \Psi)\right\|_{p} \leq\left\|f-t_{m}\right\|_{p}+C m^{h(p)} \ln (m+1)\left\|f-t_{m}\right\|_{2} .
$$

Corollary 5.2. Assume that $\Psi$ is a uniformly bounded quasi-greedy basis of $L_{2}$. Then for $2 \leq p \leq \infty$

$$
\left\|f-G_{m}(f, \Psi)\right\|_{p} \leq C m^{h(p)} \ln (m+1) \sigma_{m}(f, \Psi)_{p} .
$$

Proof. This proof goes along the lines of proof of Theorem 5.1. However, the details are different because we need to use properties of quasi-greedy bases instead of properties of Riesz bases. We use notations from the proof of Theorem 5.1 and the representation (5.1). By Lemma 5.1 and Lemma 2.6 we get for $\left\|f-S_{P}(f)\right\|_{p}$

$$
\left\|f-S_{P}(f)\right\|_{p} \leq\left\|f-t_{m}\right\|_{p}+C m^{h(p)} \ln (m+1)\left\|f-t_{m}\right\|_{2} .
$$

Using Lemma 2.6 we obtain from (5.3)

$$
\left\|S_{P}(f)-S_{Q}(f)\right\|_{p} \leq C m^{h(p)}\left(\left\|S_{P \backslash Q}(f)\right\|_{2}+\left\|S_{Q \backslash P}(f)\right\|_{2}\right) .
$$

Next, we have by Theorem 2.1

$$
\begin{gathered}
\left\|S_{Q \backslash P}(f)\right\|_{2}=\left\|S_{Q \backslash P}\left(f-t_{m}\right)\right\|_{2} \leq C_{2}(2) \sum_{n=1}^{m} n^{-1 / 2} a_{n}\left(S_{Q \backslash P}\left(f-t_{m}\right)\right) \\
\leq C \sum_{n=1}^{m} n^{-1 / 2} a_{n}\left(f-t_{m}\right)=C \sum_{n=1}^{m} n^{-1}\left(n^{1 / 2} a_{n}\left(f-t_{m}\right)\right) \\
\quad \leq C \ln (m+1) \sup _{n} n^{1 / 2} a_{n}\left(f-t_{m}\right) \leq C \ln (m+1)\left\|f-t_{m}\right\|_{2} .
\end{gathered}
$$

For the $S_{P \backslash Q}(f)$ we have

$$
\left\|S_{P \backslash Q}(f)\right\|_{2} \leq C_{2}(2) \sum_{n=1}^{m} n^{-1 / 2} a_{n}\left(S_{P \backslash Q}(f)\right)
$$




$$
\leq C_{2}(2) \sum_{n=1}^{m} n^{-1 / 2} a_{n}\left(S_{Q \backslash P}(f)\right)=C_{2}(2) \sum_{n=1}^{m} n^{-1 / 2} a_{n}\left(S_{Q \backslash P}\left(f-t_{m}\right)\right)
$$

which has been estimated in (5.9)

$$
\leq C \ln (m+1)\left\|f-t_{m}\right\|_{2} \text {. }
$$

Combining (5.7) - (5.10) we complete the proof of Theorem 5.2.

Theorem 5.3. Assume that $\Psi$ is a democratic quasi-greedy basis of $X$. Then for any $f \in X$

$$
\left\|f-G_{m}(f, \Psi)\right\|_{X} \leq C \ln (m+1) \sigma_{m}(f, \Psi)_{X}
$$

Corollary 5.3. Assume that $\Psi$ is a uniformly bounded quasi-greedy basis of $L_{p}, 1<p<\infty$. Then

$$
\left\|f-G_{m}(f, \Psi)\right\|_{p} \leq C(p) \ln (m+1) \sigma_{m}(f, \Psi)_{p} .
$$

Proof. It is known (see [2]) that a democratic and quasi-greedy basis is an almost greedy basis. Therefore, the following inequality

$$
\left\|f-G_{m}(f, \Psi)\right\|_{X} \leq C \tilde{\sigma}_{m}(f, \Psi)_{X}
$$

holds for any $f \in X$. It remains to apply Lemma 2.4 to complete the proof of Theorem 5.3. Corollary 5.3 follows from Theorem 5.3 and Proposition 2.1 .

Theorem 5.4. Assume that $\Psi$ is a uniformly bounded quasi-greedy basis of $L_{q}, 1<q<\infty$. Then for any $m$-term polynomial

$$
t_{m}=\sum_{k \in P} b_{k} \psi_{k}, \quad|P|=m
$$

we have for $q \leq p \leq \infty$

$$
\left\|f-G_{m}(f, \Psi)\right\|_{p} \leq\left\|f-t_{m}\right\|_{p}+C(p, q) m^{(1-q / p) / 2} \ln (m+1)\left\|f-t_{m}\right\|_{q}
$$

Corollary 5.4. Assume that $\Psi$ is a uniformly bounded quasi-greedy basis of $L_{q}, 1<q<\infty$. Then for $q \leq p \leq \infty$

$$
\left\|f-G_{m}(f, \Psi)\right\|_{p} \leq C(p, q) m^{(1-q / p) / 2} \ln (m+1) \sigma_{m}(f, \Psi)_{p} .
$$


Proof. This proof goes along the lines of proof of Theorem 5.2. We use notations from the proof of Theorem 5.2 and the representation (5.1). By Lemma 5.1 and Lemma 2.6 we get for $\left\|f-S_{P}(f)\right\|_{p}$

$$
\left\|f-S_{P}(f)\right\|_{p} \leq\left\|f-t_{m}\right\|_{p}+C(p, q) m^{(1-q / p) / 2} \ln (m+1)\left\|f-t_{m}\right\|_{q}
$$

Using Lemma 2.6 we obtain from (5.3)

$$
\left\|S_{P}(f)-S_{Q}(f)\right\|_{p} \leq C m^{(1-q / p) / 2}\left(\left\|S_{P \backslash Q}(f)\right\|_{q}+\left\|S_{Q \backslash P}(f)\right\|_{q}\right) .
$$

By Lemma 2.3 we estimate

$$
\left\|S_{Q \backslash P}(f)\right\|_{q}=\left\|S_{Q \backslash P}\left(f-t_{m}\right)\right\|_{q} \leq C \ln (m+1)\left\|f-t_{m}\right\|_{q}
$$

We give another proof of this bound because it will be used in estimating $\left\|S_{P \backslash Q}(f)\right\|_{q}$. We have by Proposition 2.2

$$
\begin{gathered}
\left\|S_{Q \backslash P}(f)\right\|_{q}=\left\|S_{Q \backslash P}\left(f-t_{m}\right)\right\|_{q} \leq C(q) \sum_{n=1}^{m} n^{-1 / 2} a_{n}\left(S_{Q \backslash P}\left(f-t_{m}\right)\right) \\
\leq C(q) \sum_{n=1}^{m} n^{-1 / 2} a_{n}\left(f-t_{m}\right)=C(q) \sum_{n=1}^{m} n^{-1}\left(n^{1 / 2} a_{n}\left(f-t_{m}\right)\right) \\
\leq C(q) \ln (m+1) \sup _{n} n^{1 / 2} a_{n}\left(f-t_{m}\right) \leq C(q) \ln (m+1)\left\|f-t_{m}\right\|_{q} .
\end{gathered}
$$

For the $S_{P \backslash Q}(f)$ we have

$$
\begin{gathered}
\left\|S_{P \backslash Q}(f)\right\|_{q} \leq C(q) \sum_{n=1}^{m} n^{-1 / 2} a_{n}\left(S_{P \backslash Q}(f)\right) \\
\leq C(q) \sum_{n=1}^{m} n^{-1 / 2} a_{n}\left(S_{Q \backslash P}(f)\right)=C(q) \sum_{n=1}^{m} n^{-1 / 2} a_{n}\left(S_{Q \backslash P}\left(f-t_{m}\right)\right)
\end{gathered}
$$

which has been estimated in (5.13)

$$
\leq C(q) \ln (m+1)\left\|f-t_{m}\right\|_{q}
$$

Combining (5.11) - (5.14) we complete the proof of Theorem 5.4. 


\section{Lebesgue-type inequalities II}

In this section we continue to prove Lebesgue-type inequalities for greedy approximation in $L_{p}$ under different assumptions on a basis $\Psi$. In this section we assume that $\Psi$ is a quasi-greedy basis for a pair of spaces: $L_{q}, 1<q<\infty$, and $L_{p}, q \leq p$.

Theorem 6.1. Assume that $\Psi$ is a semi-normalized quasi-greedy basis for both $L_{q}$ and $L_{p}$ with $1<q \leq 2 \leq p<\infty$. Then for any $m$-term polynomial

$$
t_{m}=\sum_{k \in P} b_{k} \psi_{k}, \quad|P|=m
$$

we have

$$
\left\|f-G_{m}(f, \Psi)\right\|_{p} \leq\left\|f-t_{m}\right\|_{p}+C(p, q) \ln (m+1)\left\|f-t_{m}\right\|_{q} .
$$

Corollary 6.1. Assume that $\Psi$ is a semi-normalized quasi-greedy basis for both $L_{q}$ and $L_{p}$ with $1<q \leq 2 \leq p<\infty$. Then

$$
\left\|f-G_{m}(f, \Psi)\right\|_{p} \leq C(p, q) \ln (m+1) \sigma_{m}(f, \Psi)_{p} .
$$

Proof. This proof goes along the lines of proof of Theorem 5.2. We use notations from the proof of Theorem 5.1 and the representation (5.1). By Lemma 5.1 and Lemma 2.7 we get for $\left\|f-S_{P}(f)\right\|_{p}$

$$
\left\|f-S_{P}(f)\right\|_{p} \leq\left\|f-t_{m}\right\|_{p}+C(p, q) \ln (m+1)\left\|f-t_{m}\right\|_{q} .
$$

We obtain from (5.3)

$$
\left\|S_{P}(f)-S_{Q}(f)\right\|_{p} \leq\left\|S_{P \backslash Q}(f)\right\|_{p}+\left\|S_{Q \backslash P}(f)\right\|_{p} .
$$

Next, we have by Theorem 2.1

$$
\begin{aligned}
& \left\|S_{Q \backslash P}(f)\right\|_{p}=\left\|S_{Q \backslash P}\left(f-t_{m}\right)\right\|_{p} \leq C_{2}(p) \sum_{n=1}^{m} n^{-1 / 2} a_{n}\left(S_{Q \backslash P}\left(f-t_{m}\right)\right) \\
& \leq C(p) \sum_{n=1}^{m} n^{-1 / 2} a_{n}\left(f-t_{m}\right)=C(p) \sum_{n=1}^{m} n^{-1}\left(n^{1 / 2} a_{n}\left(f-t_{m}\right)\right) \\
& \leq C(p) \ln (m+1) \sup _{n} n^{1 / 2} a_{n}\left(f-t_{m}\right) \leq C(p, q) \ln (m+1)\left\|f-t_{m}\right\|_{q} .
\end{aligned}
$$

For the $S_{P \backslash Q}(f)$ we have by Theorem 2.1

$$
\left\|S_{P \backslash Q}(f)\right\|_{p} \leq C_{2}(p) \sum_{n=1}^{m} n^{-1 / 2} a_{n}\left(S_{P \backslash Q}(f)\right)
$$




$$
\leq C_{2}(p) \sum_{n=1}^{m} n^{-1 / 2} a_{n}\left(S_{Q \backslash P}(f)\right)=C_{2}(p) \sum_{n=1}^{m} n^{-1 / 2} a_{n}\left(S_{Q \backslash P}\left(f-t_{m}\right)\right)
$$

which has been estimated in (6.3)

$$
\leq C(p, q) \ln (m+1)\left\|f-t_{m}\right\|_{q}
$$

Combining (6.1) - (6.4) we complete the proof of Theorem 6.1.

Remark 6.1. The statement of Corollary 6.1 holds even if we drop the assumption that $\Psi$ is quasi-greedy basis of $L_{q}$.

Proof. Assumption that $\Psi$ is semi-normalized for both $L_{q}$ and $L_{p}, q \leq 2 \leq p$, implies that it is semi-normalized in $L_{2}$. Then as in Proposition 2.1 we can prove that $\Psi$ is democratic with $\varphi(m) \asymp m^{1 / 2}$. It remains to apply Theorem 5.3 .

Now we prove sharper results for uniformly bounded orthonormal quasigreedy basis.

Theorem 6.2. Assume that $\Psi$ is a uniformly bounded orthonormal quasigreedy basis for $L_{p}, 2 \leq p<\infty$. Then for any $m$-term polynomial

$$
t_{m}=\sum_{k \in P} b_{k} \psi_{k}, \quad|P|=m
$$

we have

$$
\begin{gathered}
\left\|f-G_{m}(f, \Psi)\right\|_{p} \leq\left\|f-t_{m}\right\|_{p}+C(p) \ln (m+1)\left\|f-t_{m}\right\|_{p^{\prime}} \\
\left\|f-G_{m}(f, \Psi)\right\|_{p} \leq\left\|f-t_{m}\right\|_{p}+C(p)(\ln (m+1))^{1 / 2}\left\|f-t_{m}\right\|_{2} .
\end{gathered}
$$

Corollary 6.2. Assume that $\Psi$ is a uniformly bounded orthonormal quasigreedy basis for $L_{p}, 2 \leq p<\infty$. Then

$$
\left\|f-G_{m}(f, \Psi)\right\|_{p} \leq C(p)(\ln (m+1))^{1 / 2} \sigma_{m}(f, \Psi)_{p} .
$$

Proof. By Theorem $2.2 \Psi$ is a quasi-greedy basis of $L_{p^{\prime}}$. Thus, (6.5) follows from Theorem 6.1 with $q=p^{\prime}$. We now prove (6.6). As in the proof of Theorem 6.1 we obtain by Lemma 5.1 and Lemma 2.8

$$
\left\|f-S_{P}(f)\right\|_{p} \leq\left\|f-t_{m}\right\|_{p}+C(p)(\ln (m+1))^{1 / 2}\left\|f-t_{m}\right\|_{2} .
$$


By Theorem 2.1 we obtain

$$
\left\|S_{Q \backslash P}(f)\right\|_{p}=\left\|S_{Q \backslash P}\left(f-t_{m}\right)\right\|_{p} \leq C(p) \sum_{n=1}^{m} n^{-1 / 2} a_{n}\left(f-t_{m}\right)
$$

$$
\leq C(p)\left(\sum_{n=1}^{m} n^{-1}\right)^{1 / 2}\left(\sum_{n=1}^{m} a_{n}\left(f-t_{m}\right)^{2}\right)^{1 / 2} \leq C(p)(\ln (m+1))^{1 / 2}\left\|f-t_{m}\right\|_{2} .
$$

As in the proof of Theorem 6.1 we get

$$
\left\|S_{P \backslash Q}(f)\right\|_{p} \leq C(p) \sum_{n=1}^{m} n^{-1 / 2} a_{n}\left(f-t_{m}\right)
$$

and by the intermediate step in (6.8)

$$
\leq C(p)(\ln (m+1))^{1 / 2}\left\|f-t_{m}\right\|_{2} .
$$

It remains to use representation (5.1) and inequality (6.2).

If $\Psi$ is assumed to be uniformly bounded, then the Lebesgue-type inequality of Theorem 6.1 holds whenever $q \leq p$.

Theorem 6.3. Assume that $\Psi$ is a uniformly bounded quasi-greedy basis for both $L_{q}$ and $L_{p}$ with $1<q \leq p<\infty$. Then for any $m$-term polynomial

$$
t_{m}=\sum_{k \in P} b_{k} \psi_{k}, \quad|P|=m
$$

we have

$$
\left\|f-G_{m}(f, \Psi)\right\|_{p} \leq\left\|f-t_{m}\right\|_{p}+C(p, q) \ln (m+1)\left\|f-t_{m}\right\|_{q}
$$

Proof. As in the proof of Theorem 6.1 we obtain by Lemma 5.1 and Lemma 2.9

$$
\left\|f-S_{P}(f)\right\|_{p} \leq\left\|f-t_{m}\right\|_{p}+C(p, q) \ln (m+1)\left\|f-t_{m}\right\|_{q}
$$

By Proposition 2.2 we obtain

$$
\begin{aligned}
& \left\|S_{Q \backslash P}(f)\right\|_{p}=\left\|S_{Q \backslash P}\left(f-t_{m}\right)\right\|_{p} \leq C(p, q) \sum_{n=1}^{m} n^{-1 / 2} a_{n}\left(f-t_{m}\right) \\
& \quad \leq C(p, q) \sum_{n=1}^{m} n^{-1}\left\|f-t_{m}\right\|_{q} \leq C(p, q) \ln (m+1)\left\|f-t_{m}\right\|_{q} .
\end{aligned}
$$


As in the proof of Theorem 6.1 we get

$$
\left\|S_{P \backslash Q}(f)\right\|_{p} \leq C(p, q) \sum_{n=1}^{m} n^{-1 / 2} a_{n}\left(f-t_{m}\right)
$$

and by the intermediate step in (6.10)

$$
\leq C(p, q) \ln (m+1))\left\|f-t_{m}\right\|_{q}
$$

It remains to use representation (5.1) and inequality (6.2).

\section{Acknowledgements}

The first author was a participant in the NSF supported Workshop in Analysis and Probability at Texas A\&M University in 2011 and his research was supported by NSF grant DMS-1101490. The research of the second author was supported by grants MTM2010-15790 and FPU of Universidad Autónoma de Madrid (Spain). The research of the third author was supported by NSF grant DMS-0906260. The second author wishes to thank the Department of Mathematics of the University of South Carolina for hospitality during his stay in fall 2011, and specially Prof. S. J. Dilworth and Prof. V. N. Temlyakov.

\section{References}

[1] S. J. Dilworth, N. J. Kalton, and Denka Kutzarova, On the existence of almost greedy bases in Banach spaces, Studia Math. 15 (2003), 67-101.

[2] S. J. Dilworth, N. J. Kalton, Denka Kutzarova, and V. N. Temlyakov, The Thresholding Greedy Algorithm, Greedy Bases, and Duality, Constr. Approx. 19 (2003), 575-597.

[3] S. J. Dilworth, M. Soto-Bajo, and V. N. Temlyakov, Quasi-greedy bases and Lebesgue-type inequalities, IMI Preprint 2012:02, 2012.

[4] V. F. Gaposhkin, On unconditional bases in $L^{p}$-spaces, Uspekhi Mat. Nauk 13 (1958), 179-184.

[5] Ulf Grenander and Gabor Szegö, Toeplitz forms and their applications, Univ. of California Press, Los Angeles, CA, 1958. 
[6] A. Grothendieck, Résumé de la théorie métrique des produits tensoriels topologiques, Bol. Soc. Mat. Sao Paulo 8 (1953/1956), 1-79.

[7] E. Hernández, Lebesgue-type inequalities for quasi-greedy bases, arXiv:submit/0349580, 2011.

[8] K. S. Kazarian, On the logarithmic growth of the arithmetic means of the sums of Lebesgue functions of bounded biorthogonal systems, Doklady AN Arm. SSR 69 (1979), 140-145 (Russian).

[9] S. V. Konyagin and V. N. Temlyakov, A remark on greedy approximation in Banach spaces, East. J. Approx. 5 (1999), 365-379.

[10] S. V. Konyagin and V. N. Temlyakov, Greedy Approximation with Regard to Bases and General Minimal Systems, Serdica Math. J. 28 (2002), 305-328.

[11] S. Kostyukovsky and A. Olevskii, Note on decreasing rearrangement of Fourier series, J. Appl. Anal. 3 (1997), 137-142.

[12] H. Lebesgue, Sur les intégrales singuliéres, Ann. Fac. Sci. Univ. Toulouse (3) 1 (1909), 25-117.

[13] J. Lindenstrauss and L. Tzafriri, Classical Banach Spaces II, Springer, Berlin, 1979.

[14] Bernard Maurey, Isomorphismes entre espaces $H_{1}$, Acta Math. 145 (1980), 79-120.

[15] Morten Nielsen, An example of an almost greedy uniformly bounded orthonormal basis for $L_{p}(0,1)$, J. Approx. Theory 149 (2007), 188192.

[16] A. M. Olevskii, On an orthonormal system and its applications, Mat. Sb. 71(113) (1966), 297-336; English transl. in Amer. Math. Soc. Transl. (2) 76 (1968).

[17] A. M. Olevskii, Fourier Series with Respect to General Orthonormal Systems, Springer, Berlin, 1975.

[18] R. E. A. C. Paley, On the lacunary coefficients of power series, Ann. Math. 34 (1933), 615-616. 
[19] G. Pisier, Factorization of linear operators and geometry of Banach spaces, CBMS 60, Amer. Math. Soc, Providence R.I., 1986.

[20] M. M. Popov, A property of convex basic sequences in $L_{1}$, Methods Funct. Anal. Topology 11 (2005), 409-416.

[21] S. J. Szarek, Bases and biorthogonal systems in the spaces $C$ and $L^{1}$, Ark. Mat. 17 (1979), 255-271.

[22] V. N. Temlyakov, Greedy Algorithm and $m$-Term Trigonometric Approximation, Constr. Approx. 14 (1998), 569-587.

[23] V. N. Temlyakov, Nonlinear method of approximation, Found. Compt. Math. 3 (2003), 33-107.

[24] V. N. Temlyakov, Greedy approximation, Acta Numerica 17 (2008), 235-409.

[25] V. N. Temlyakov, Greedy approximation, Cambridge University Press, 2011.

[26] V. N. Temlyakov, M. Yang, P. Ye, Greedy approximation with regard to non-greedy bases, Adv. Comput. Math. 34 (2011), 319-337.

[27] V. N. Temlyakov, M. Yang, P. Ye, Lebesgue-type inequalities for greedy approximation with respect to quasi-greedy bases, East J. Approx. 17 (2011), 127-138.

[28] P. Wojtaszczyk, Greedy Algorithm for General Biorthogonal Systems, J. Approx. Theory 107 (2000), 293-314. 